\title{
INTRODUCTORY REPORT
}

\section{THE RECENT DEVELOPMENT OF RISK THEORY AND ITS APPLICATIONS \\ J. KUPPER \\ Zürich}

The response to the invitation for the 5 th Colloquium has been, especially for subject I, very gratifying; a large number of meritorious papers have been sent in. Fortunately, it took not much time in this case to find a good guide for all this work. R. E. Beard presents in his article "Some Actuarial Aspects of Non-Life Insurance Company Management" 1 ) a broad cross-section of the many problems that confront the non-life insurer. It was therefore regarded as not quite unwise to follow his steps and to discuss the other papers at suitable points.

In the first half of his work R. E. Beard deals with a practical example from motor insurance, which is fully calculated with the help of numerical data. The question relates to the estimation of the value of outstanding claims, a quantity which is of decisive importance for the evaluation of the results of a portfolio. Apart from chance fluctuations which might affect the statistics from year to year, there are three major factors that render the task of estimation difficult:

(a) the natural growth of the portfolio

(b) the fact that bigger claims require longer time for settlement than the smaller ones

(c) the change in the value of money with time.

In order to reach the goal set, the following particulars of several successive periods of account are necessary:

the number of claims intimated, the number of claims settled and the amounts of claims settled.

1) This article has been enclosed in Vol. IV Part I. 
The model on which the author bases his calculations emanates from the following assumptions:

(A) the number of intimated claims increase each year by $50 \%$

(B) the distribution of the amount of claims can be represented by an exponent polynomial

(C) the value of money experiences a decrease of about $\mathrm{II} \%$ per year

(D) the period of settlement can be represented by exponential expressions with negative indices.

On the basis of the model described just now, R. E. Beard calculates the data mentioned before, which are essential for the determination of outstanding claims. These data are his "observed" values. By his method the stochastic fluctuations which would otherwise characterise the basic data are diminished. Partly this is to be regretted since the real value of the study is to a certain extent lowered thereby: on the other hand it is presicely because of this method that the other influences can be so exactly and pointedly analysed as would otherwise be the case. Considering that here it is more in the nature of a striking illustration, this point of view does offer its own advantages. The author then shows how the given statistical data should be rectified in order to give due consideration to the disturbing influences of growth, inflationary trend and time lag to settle. This leads finally to the goal mentioned at the start- to the estimation of outstanding claims.

The second section of R. E. Beard's essay deals with the question of rating. At first the importance of a fair risk premium is underlined. The possible consequences of a less suitable premium structure are shown by a well selected hypothetical example.

The need for a scientific treatment of the question is imperative especially here. In this respect, the author commends J. Mehring's paper in the "Blätter" (Oct. I964) with its modern statistical methods. Opinions differ as to how the tariffs should be fixed and to what criteria should regard be had in order to realise a fair premium. At the last congress in Trieste, P. S. Delaporte has given an interesting solution with the help of the so-called "prime modelée sur le risque". 
In order to derive correct premiums for an insurance portfolio it is essential to have the knowledge of the underlying claim distributions. At this point we may now tackle the first important class of problems that are to be dealt with in the present Colloquium.

\section{The evaluation of the distribution function of total claims}

The probability that the total amount of claims is $\leqslant x$ is given in the usual notation by the distribution function

$$
F(x, t)=\sum_{n=0}^{\infty} P_{n}(t) S^{* n}(x)
$$

For the distribution of the number of claims $P_{n}(t)$ assumption is very frequently made that

$$
P_{n}(t)=\int_{0}^{\infty} e^{-\lambda t} \frac{(\lambda t)^{n}}{n !} d U(\lambda)
$$

where the two standard cases for the structure function $U(\lambda)$ are given by

$$
U(\lambda)=\varepsilon(\lambda-\mathrm{I}) \quad(\rightarrow \text { Poisson })
$$

and

$$
U(\lambda)=\frac{I}{\Gamma(k)} \int_{0}^{\lambda k} e^{-y} y^{k-1} d y \quad(\rightarrow \text { negative binomial })
$$

However simple the determination of the moments of the distribution of total claims may be, the difficulty of calculating the distribution function $F$ itself is considerable. Even for elementary functions, the formulae are rather complicated owing to the $n$th order convolution. From the very early stage, therefore, one has been on the lookout for approximate solutions. I should like to point out here specifically the excellent and detailed survey available in the article by $\mathrm{H}$. Bohman and F. Esscher in the SAT 1963. Apart from theoretical investigation, voluminous statistical and numerical data have been treated in this article.

E. Pesonen in his contribution "On the calculation of the generalised Poisson function" points out further possibilities for the 
calculation of the total claim distribution, here, in particular, for

$$
F(x, t)=e^{t} \sum_{n=0}^{\infty} \frac{t^{n}}{n !} S^{* n}(x)
$$

His suggestions are as follows:

(A) In order to determine the function $F(x, t)$, resort may be had to the well-known Esscher-transformation. The author's first proposal consists in a slight modification of this often used method. The transformation parameter $h$ in the defining equation is now supposed to be a variable too. If the number of terms taken into consideration is sufficiently large, this modified Esscher method can lead to the same result as the expansion in the next section (B).

(B) The distribution function of the amount of claims can very often be adequately represented by exponent polynomials. As mentioned before, R. E. Beard, too, has made avail of such a model. If the function consists of $k$ components, then the corresponding distribution of total claims can also be formed out of $k$ components, which must be convoluted with one another. For one component, $S(x)=\mathrm{I}-e^{-a x}$, an approximation method may be selected which makes use of the asymptotic properties of Bessel-functions. In this special case, the density of total claims for $x>0$ is equivalent to

$$
f(x, t)=\sqrt{\frac{t a}{x}} e^{-(a x+t)} I_{1}(2 \sqrt{t a x})
$$

This possibility also offers auxiliary means for getting quickly a rough picture of the distribution function $F(x, t)$, in case the distribution of the amount of claims does not deviate too much from the exponential distribution.

(C) The distribution $S(x)$ is approximated by a step function, permitting the total claims to be represented as convolution of simple Poisson distributions. Instead of the function itself, upper and lower limits are calculated.

(D) A random sample, originating from a stochastic variable with distribution function $F(x, t)$, is obtained by simulation. The random sample then serves directly as an estimation of the distribution function. These Monte Carlo techniques have found entry in various branches of economics in recent years. 
(E) In the next section E. Pesonen introduces a mixed procedure. Here "mixed" signifies that various methods may be applied to the individual components of the distribution function $F$. Let $F=F_{1} * F_{2} * F_{3}$, where $F_{1}$ comprehends the claims $\leqslant \xi, F_{2}$ the ones between $\xi$ and $\eta$, and $F_{3}$ the ones $>\eta$. Then, $F_{1}$ can be approximated by a normal distribution, whereas for $F_{2}$ and $F_{3}$ the Monte Carlo method may be envisaged. To select the points $\xi$ and $\eta$ as well as possible is of great importance.

(F) Finally, it has been pointed out that it would be a material simplification of the problem, if a distribution function could be found, which always remains below the original function, which is thus "more dangerous". The general proposition put forward by the author can be proved in a special case, but the question of general validity remains open.

The paper of E. Pesonen offers a good deal of stimulus and deserves to be pursued further.

In close connection with the topic of this paper is the next one of $E$. Hovinen "A precedure to compute the values of the generalised Poisson function". The author gives some more details of the method marked above with (E) and uses it to set up a computer program for the calculation of the distribution function wanted. This program is explained thoroughly and written down in full for an Elliott 503 Computer in ALGOL language. According to the experience of the author this method combines the necessary requirements of accuracy with computational rapidness. Some possible modifications and improvements of the procedure are indicated. As E. Hovinen made it known in the discussion, the committee in Finland that undertook the study of these problems has not yet finished its work. However, numerical figures should also be available soon, and it will certainly be interesting to look at the published results.

The weighty work of B. Almer "Modern General Risk Theory; On the Road from Risk Elements to Poisson and from Risk Statistics to the Exact Distribution of Total Claim Amount" investigates the same problem in its last section.

A first solution has been already presented by the author in 
New York 1957. Systematic investigations have shown that an exponent polynomial with 3 or 4 components is adequate to represent the claims amount curve. Hence, the resultant solution is an asterisk multiplication of 4 similar Bessel functions. The author's second solution utilises the asymptotic expansion of Hankel functions. The application of this formula to the original elementary solution (solution for one component) permits representation in the form of a product of a strict normal term and a set of short series. An addendum gives some further explanations of the procedure. Here too, relations to E. Pesonen's essay, part (B), should be borne in mind.

But the paper of B. Almer is not confined to this special problem; it contains an intensity of other thoughts and gives a perspicuous insight in the fundamentals of risk theory in a very general manner. The first part deals with the brickworks of this theory-the risk elements-characterised by claims probability, claims curve and contribution to total claims amount. Risk systems or sets are formed out of risk elements. The author analyses their properties and explains various expressions and symbols. Then, three error theorems are derived.

The first one is already contained in the New York paper of the author. The purport is that all individual risk systems satisfying a well-defined condition are determined by two limits, a system with maximum symmetry (homogenized system) and a system with maximum asymmetry (reduced homogenized system). A good estimate for the degree of inhomogeneity is shown to be important; a valuable approach for this is the study of the accumulating curve.

The second error theorem deals with the relative errors of statistical quantities, in case the risk system has converged to Poisson. For the a posteriori number of claims $v$ with an a priori value $n$, and consequently also for the claims frequency, the corresponding formulae have been known for a long time, while the analogous formulae for the amount of total claims $\nu$ with a priori value $y$, and hence for the risk premium also, are a modern result. The third error theorem is, finally, analysed taking into account the uncertainty of prognosis.

The second part of B. Almer's paper establishes the mutual connection between risk theory and probability theory. It turns 
out that the standard definitions of modern probability theory cannot be straight away transcribed in risk theory. In this connection, the third error theorem proves to be important. With the definition of stochastic disorder, various facts can be stated in form of theorems.

Furthermore, inhomogeneous risk groups have been closely examined. The concept of risk lines is introduced with two possible systems, the branch form and the preselection form. With the help of these expressions a uniformisation theorem can be formulated.

G. Andreasson's article "Distribution Free Approximation in Applied Risk Theory" ') continues the thought process originated by $\mathrm{H}$. Bühlmann at the Trieste Colloquium two years ago. Bühlmann then directed attention to the fairly arbitrary assumptions often made regarding the distribution function of the number of claims and the amount of claims and emphasised that for many problems the knowledge of the first two moments of the distribution was sufficient. His aim was to obtain estimates of these moments on a distribution free basis. For G. Andreasson the estimate of moments is merely the means to the goal of computing the distribution function $F$.

The starting point for the consideration differs somewhat from the classic structure of the model indicated before. G. Andreasson proceeds from the standpoint of individual risks, that is, he investigates the probability that I policy produces $n_{1}$ claims during the observed time interval. If the risk group consists of $N$ independent policies and

$$
n=n_{1}+n_{2}+\ldots+n_{N}
$$

denotes the total number of all the claims of the group, then, in addition, for the distribution of the number of claims one has to take an $N$-fold convolution into account. For the two standard examples, Poisson and Polya, this presents no difficulties whatsoever.

$\mathrm{H}$. Ammeter has already shown in his work published in the SAT I948, which has subsequently proved to be epoch-making, that in Polya case for $N \rightarrow \infty$ the distribution of the standardised variable of total claims $z$ tends to the normal distribution $\Phi(z)$. For large values of $N$, the asymptotical expansion

1) Published in Astin Bulletin Vol. IV part I. 


$$
F(z, N t)=\Phi(z)-\frac{\gamma}{3 !} \Phi^{(\prime \prime \prime)}(z)
$$

may be used, where $\gamma$ has the usual meaning. The author makes the assumption that this relation holds good even where the population of the number of claims is not exactly distributed according to Polya (Poisson). The principal task then consists in estimating the moments contained in $z$ and $\gamma$. This can be done, as mentioned before, without any knowledge of the underlying distribution, on the basis of the empirical moments alone. The expressions for the mean and for the variance agree with those given formerly by $\mathrm{H}$. Bühlmann.

In order to compare the merit of the method, the author has on the one hand calculated the distribution function of total claims for statistical data from the Swedish third party motorcar insurance in accordance with the distribution free method described. On the other hand, the observed values have been fitted with a typical standard model (distribution of the number of claims = Polya; distribution of the amount of claims $=$ exponent polonomial with 4 components), and the distribution function has been calculated by the Esscher method. At least, for large values of $N$, there exists no material difference between the two methods. This result is a valuable one, but not really surprising.

The next chapter of R. E. Beard's paper deals with some of the problems of fire insurance. The present report shall not enter into this subject, since the topic is not supposed to be discussed in detail at this colloquium.

In a further section, the author comes to the discussion of reinsurance. The trend towards forms of non-proportional cover is brought into prominence. Non-proportional reinsurance is essentially based on the hypothesis that claim amounts can be regarded as random samples out of a population with known distribution function. Only then the calculation of reinsurance premiums is possible. Loading by considerable margins cannot be helped, generally, to provide for possible high fluctuations.

The interest of the actuaries in this field finds expression in the number of papers published year after year; even here 4 contributions have been presented. 
Reinsurance problems

By the definition given earlier for the distribution function $F(x, t)$, the net premium for a stop loss reinsurance that covers the amount of claims by which the total amount may exceed $x$, is expressed by the formula

$$
\pi(x, t)=\int_{x}^{\infty}(z-x) d F(z, t)
$$

and the corresponding variance

$$
\sigma_{\pi}^{2}=\int_{x}^{\infty}(z-x)^{2} d F(z, t)-\pi^{2}
$$

It is to be observed that this variance is smaller than the variance reduction experienced by the cedent through surrender of his part portfolio (Vide Borch at the r6th Congress in Brussels, I960).

F. Esscher, in his article "Some Problems Connected with the Calculation of Stop Loss Premiums for large Portfolios", has recourse to Ove Lundberg's theorem that, assuming $\int_{0}^{\infty} x d S(x)=$ $\mathrm{s}_{1}=\mathrm{I}$, the distribution function of total claims $F(s t, t)$ tends to the structure distribution $U(s)$ for large values of $t$. Analogous limit considerations can be envisaged for the premium and the variance.

The author initiates three investigations:

(A) On extremely variegated reasons (to take into account, for example, heterogeneity, contagion, cumulative claims), the $\Gamma$-function has been used for the structure function. This choice is however not peremptory; other functions may probably give better results. Theoretically, various alternatives have been put forward already, including e.g. in an article of the rapporteur ("Blätter" I962). It is very creditable that the author has for once indicated in terms of figures the effects of various structure functions to be investigated.

The resulting differences between the various methods appear to be surprisingly small, especially in the neighbourhood of $s=\mathbf{I}$. If, as usual, a loading of an $\varepsilon$-multiple of the standard deviation on the net premium is consequently suggested, then the influence 
exerted on the premium by the choice of the structure distribution appears to be of slight importance.

In view of these considerations the author continues his work on the basis of the classic hypothesis.

(B) For the difference $\frac{\pi(s t, t)}{t}-\lim _{t \rightarrow \infty} \frac{\pi(s t, t)}{t}$ an asymptotical representation has been derived. Even here numerical tables are given. They show that for large values of $t$ the mistake made by calculating with the limit instead of $\pi(s t, t) / t$ is of little weight.

(C) In the last part, F. Esscher comes across another interesting question. The hypothesis put forward is that the portfolio can be broken down into 2 (or more) subgroups, independent of each other. H. Ammeter has already (in the "Mitteilungen" of the Swiss Actuaries, 1957) paid attention to such a division of the risk aggregate into subgroups, having regard to excess claims and ruin.

By generalisation of Lundberg's theorem it is possible to determine with the help of characteristic functions the limiting value of $F\left(s t s_{1}, t\right)$, when $t \rightarrow \infty$. The calculation of the limit value for the net premium of stop loss reinsurance has been obtained with the help of the transformation named after the author. And here numerical values have been tabulated under various assumptions. For example, it is very gracefully shown how through the division of a portfolio into 2 subgroups, where for each subgroup the expected total claims amount to $50 \%$ of the total claims of the undivided group, the stop loss premium (for the same $k$ in each subgroup) reduces sharply. On the other hand it appears that a stop loss cover that comprises all the independent groups in one lot is to be preferred to the individual coverage of these several groups.

Other two contributions deal with optimum properties.

E. Pesonen raises again the problem of the optimum reinsurance form in his article "On Optimal Properties of the Stop Loss Reinsurance". Borch, in his work referred to earlier, and later on Kahn (ASTIN-Bulletin r96I) in a more general form have shown that the stop loss reinsurance is most efficient in the sense that it keeps the variance of the part retained by the cedent to a minimum. The present note gives a rather different, elegant proof of this fact with the help of conditional expected values as defined by Doob. 
Great interest deserves the paper of H. G. Verbeek "On Optimal Reinsurance". ${ }^{1}$ ) By conclusion of a reinsurance contract the cedent desires to attain a certain stability for his portfolio at lowest cost. The variance of the part of risk retained can adequately be regarded as a measure of stability. The author assumes that a reinsurance policy is effected in accordance with a surplus system with retention $U$. By increasing this retention the costs of the cedent can be reduced, but then of course allowance must be made for a smaller stability. The question therefore arises whether the earlier stability may not be reattained by an appropriate stop loss reinsurance, keeping nevertheless the costs below the earlier level. In a certain sense even this work is a continuation of the thought of Borch who, as just stated, has shown that the stop loss, within the meaning of maximum stability, represents the most effective form of reinsurance. However, he ignores the loading on premiums, a matter which from the practical point of view, especially in stop loss contracts, should not be overlooked. It is therefore not surprising that, in consideration of this fact, a pure stop loss reinsurance does not represent the cheapest solution of the problem.

The author resorts to the usual model, number of claims Poisson distributed by mean $t$, distribution of the amount of claims $S(x)$. If a surplus reinsurance is in force, it is equivalent to a truncation of the distribution function $S(x)$ at point $u$, the probability measure of the interval $(u, \infty)$ being concentrated at point $u$. For the computation of total claims the variable $x$ is standardised and the Edgeworth expansion is applied.

The stop loss reinsurance which is now required to be effected takes into account the mean claim $t s_{1}(u)$ of the total claims distribution. All claims in excess of the limit $v t s_{1}(u)$ are covered by the reinsurer. Net stop loss premium and variance are calculated with the help of the transformation and expansion mentioned above; then the moment condition about stability stated before is formulated and discussed. A main result is that a stop loss reinsurance cannot reduce the variance to less than approximately $\mathrm{I} / 3$.

The costs of reinsurance are composed of two parts:

(a) Total profit margin $\gamma$, which will be ceded on retention $u$.

1) Published in ASTIN Bulletin Vol. IV part I. 
(b) Loading for net stop loss premium, a fraction $\varepsilon<\mathrm{I}$ of the standard deviation of this premium.

The author calculates the minimum of this function of $u$ and $v$ under the auxiliary condition of constant stability. It is relatively easy to show that neither a pure stop loss reinsurance $(u=\infty)$, nor a mere surplus form $(v=\infty)$ represents the minimum, but a combination of both. An illustrative numerical example of the theory is given with $\gamma=5 \%$ and $\varepsilon=50 \%$ and a gamma distributed claim amount.

In this connection, the somewhat belated paper of G. Benktander and J. Ohlin "A Combination of Surplus and Excess Reinsurance of a Fire Portfolio" is very interesting too: here, also, two reinsurance systems are joined. G. Benktander emphasised in his personal comments the importance of optimum reinsurance properties, but the present paper is merely thought as a preliminary note concerning the interplay between the two premiums $R(M)$ and $\pi_{M}(m)$. The first means the ceded risk premium volume on surplus basis with a PML-retention $M$, the second the corresponding excess of loss premium with a priority $m$ and the just-mentioned surplus retention $M$. Some simple attributes of the two functions can be derived without difficulties. For a more accurate study something about the claims amount distribution must be known. With two rather specific assumptions, this distribution turns out to consist of a uniformly distributed part over the interval $(0, a)$ and a Pareto-distributed part over $(a, \infty)$, where $a$ signifies the truncation point of the Pareto distribution. Now the quantities $R(M), \pi_{M}(m)$ and their sum, the total volume of risk premiums ceded on both systems, are calculated by relatively simple formulae. An illustrative numerical example concludes the note.

The last section" of R. E. Beard's survey is concerned with "accounts and statutory returns". The thoughts contained in it enter, in particular, the realm of subject 3 . One of the material questions in this connection is that of the possible ruin of the insurer. A paper dealing with a more theoretical aspect has been kept for discussion in the section of risk theory.

In "The Random Walk of a Simple Risk Business" 1), H. L. Seal

1) Published in ASTIN Bulletin Vol. IV Part I. 
reproduces in a succinct form the contents of a lecture delivered at his university.

A simple risk process is defined as a business with only one type of contract under which, in consideration of payment of a unit premium, an insured sum $m$ ( $m$ integral) becomes payable on the happening of the claim event. After a historical sketch of the hitherto existing possibilities of getting hold of the problem, the author follows up the method known from sequential random sampling. The process is represented as a random walk along the $x$-axis, beginning with a positive value (initial reserve). If no claim event occurs, a step is taken towards right (with probability $p$ ); if it occurs, $m$ steps are taken towards left (with probability $q$ ). The process is supposed to have the upper limit $M$, which means that as from the moment the risk reserve amounts to $M$ no further premiums have to be paid. On the other hand, ruin results if at any moment point zero is reached or crossed. Two probabilities may be distinguished here:

(a) $v_{x, n}$

(b) $v_{x}=\sum_{n=1}^{\infty} v_{x, n}$
$=$ probability that with given reserve $x$ ruin occurs on the $n$th step

$=$ probability that with given reserve $x$ ruin occurs at any moment whatever.

The simpler case b) with $m=2$ has been treated by Feller in terms of the theory of games. The probability $u_{x}=\mathrm{I}-v_{x}$ obeys a difference equation with $m$ boundary conditions. To solve this system of equations, two methods are given. For large values of $x$, the approximate solution for $u_{x}$ is

$$
u_{x} \sim C_{1}+C_{2} \lambda^{x}
$$

where $\lambda$ signifies the positive root $\neq \mathrm{I}$ of the equation

$$
p z^{m}-z^{m-1}+q=\mathrm{o}
$$

$C_{1}$ and $C_{2}$ are determined according to the boundary conditions for $x=0, M$.

Feller's method of generating functions gives a somewhat more accurate asymptotic result.

Case a) can be treated by similar considerations. 
The author underscores his exposition with several numerical quantities, which permit a better picture to be drawn of the order of magnitudes of the corresponding values.

Finally, it must be said that the submitted papers contain an intensity of thought, enriching our field of science in many directions. Regrettably, it was only possible to reproduce them incompletely; but we hope that this report will provide at least some stimulation for further study. 